\title{
Outcomes of Combined iStent Trabecular Micro-Bypass and Cataract Surgery for the Treatment of Open-Angle Glaucoma in a Saudi Population
}

This article was published in the following Dove Press journal:

Clinical Ophthalmology

\author{
Ahmed Al Habash (iD) \\ Omar Khan ${ }^{2}$ \\ 'Department of Ophthalmology, College \\ of Medicine, King Fahd Hospital of the \\ University, Imam Abdulrahman Bin Faisal \\ University, Dammam, Saudi Arabia; \\ ${ }^{2}$ Ministry of Health, Madinah, Saudi \\ Arabia
}

Purpose: To assess the effectiveness and safety of a single iStent trabecular micro-bypass stent implantation in combination with cataract surgery in lowering the intra ocular pressure (IOP) and number of anti-glaucoma medications in Saudi patients with open-angle glaucoma (OAG).

Setting: King Fahad Hospital of the University, Al Khobar, Saudi Arabia.

Design: Prospective, non-comparative, uncontrolled, non-randomized interventional case series.

Methods: One iStent was implanted during phacoemulsification cataract surgery for adult patients with OAG by a single surgeon over a period of 3 years. Main outcome measures include number of anti-glaucoma medications, IOP, corrected distance visual acuity (CDVA) and complications.

Results: Thirty-six eyes of 33 patients with OAG underwent the surgery. The pre-operative mean IOP and mean anti-glaucoma medications were $17.47 \pm 5.44 \mathrm{mmHg}$ and $2.69 \pm 0.92$, respectively. At 1 year of follow-up mean, IOP decreased to $13.44 \pm 1.99$ (23.1\% reduction) $(\mathrm{P}<0.001)$ and mean anti-glaucoma medications decreased to $1.47 \pm 1.13$ (45.4\% reduction) $(\mathrm{P}<0.001)$.

Conclusion: After 12-month follow-up, phacoemulsification with the implantation of a single iStent seems to be an effective and safe procedure to lower IOP and the number of anti-glaucoma medications, and to overcome the problems associated with medication nonadherence in OAG patients with cataracts.

Keywords: open-angle glaucoma, minimally invasive glaucoma surgery, Schlemm's canal, iStent trabecular micro-bypass

\section{Introduction}

Cataract and glaucoma are the first and second leading causes of blindness around the world. As of 2010, approximately 60 million people suffered from glaucoma, and this figure is expected to increase to 79.6 million by $2020{ }^{1}$ Similar to the rest of the world, both of these conditions are leading causes of blindness in the Kingdom of Saudi Arabia. ${ }^{2}$

Open-angle glaucoma (OAG) impairs the retinal ganglion cells. Current treatment options aim to preserve the integrity of the optic nerve and visual function by reduction of intraocular pressure (IOP). ${ }^{3}$ This was evidenced by clinical trials
Correspondence: Ahmed Al Habash PO Box 31010, Khobar 31952, Saudi Arabia

Tel +966507017777

Email ahmedalhabash@gmail.com 
demonstrating a decrease in glaucoma progression risk by around $10 \%$ for every $1 \mathrm{mmHg}$ IOP reduction. ${ }^{4}$ Treatment options include hypotensive eye drops, laser trabeculoplasty, and surgical interventions (e.g., incisional glaucoma interventions). While each is associated with positive outcomes, all have some disadvantages that can decrease their utility. ${ }^{5}$ As a case in point, adherence to prescribed topical medications is a known challenge. Potential issues such as allergies, cost, and inaccessibility may prevent the proper use of these medications. ${ }^{6}$

In the case of laser trabeculoplasty, while it is usually safe, the outcomes may not be long term. ${ }^{7,8}$ Although incisional glaucoma surgeries can effectively lower IOP, there remain real concerns to both patients and surgeons of the possible lifelong complications of the surgeries as hypotony, bleb leaks or fibrosis, or more devastating vision-threatening complications like bleb related infection and endophthalmitis. Therefore, it is not preferred for early glaucoma stage but reserved for more advanced disease. ${ }^{9}$

More recently, interest has increased in the minimallyinvasive glaucoma surgery (MIGS) devices and their abilities to reduce the IOP and medications with less serious side effects. ${ }^{10}$ Advantages of MIGS devices are the lower rate of complications, the ability to be combined with other MIGS procedures, the possibility of future incisional glaucoma surgery as it spares the conjunctiva. ${ }^{11}$

The iStent (Glaukos Corporation, San Clemente, California) is a MIGS device and the first ab-interno glaucoma implant that received FDA approval for the management of mild-to-moderate OAG. ${ }^{12}$ It works on the main pathology of high IOP in open-angle glaucoma which is the resistance of aqueous outflow through the trabecular meshwork. ${ }^{13,14}$ The iStent creates a bypass through the trabecular meshwork to Schlemm's canal, improving aqueous outflow and resulting in IOP lowering. ${ }^{15}$ This enhancement of the outflow has been identified in the literature using fluorophotometric experiment. $^{16}$

Many studies have reported significant IOP and medication reduction following iStent implantation with an excellent safety profile. ${ }^{17-23}$ In most cases, the iStent trabecular micro-bypass stent is implanted in patients with mild-to-moderate OAG. ${ }^{17,18,20-22}$ However other studies have evaluated it's usage as a treatment for both pseudoexfoliative $^{19}$ and advanced glaucoma. ${ }^{23}$

To the best of our knowledge, no previous study has evaluated the application of the iStent procedure in a patient population from the Middle East. In light of this gap in the existing literature, it is the objective of this study to assess the effectiveness of a single iStent trabecular micro-bypass stent implantation in combination with cataract surgery in Saudi patients.

\section{Patients and Methods \\ Study Design}

The present study is a prospective, non-comparative, uncontrolled, non-randomised interventional case series of patients who underwent combined phacoemulsification with one iStent implantation. All the surgeries were performed by a single surgeon (A.H.) over the course of 3 years at King Fahad Hospital of the University in Khobar, Saudi Arabia. The inclusion criteria included patients from Saudi origin who had cataract with OAG, completion of the phacoemulsification surgery without intraoperative complications, usage of any number of pre-operative glaucoma drugs. The exclusion criteria included; other forms of glaucoma other than OAG, cloudy cornea, since this undermines gonioscopic angle view; peripheral anterior synechiae, active uveitis, and coexisting retinopathy that limits visual acuity potential. The study followed the tenets of the Declaration of Helsinki and was approved by the hospital's institutional review board. Written informed consent was obtained from all patients before surgery.

Pre-operative data included gender, age, diagnosis, angle status, number of anti-glaucoma medications, cupto-disc ratio, IOP, and corrected distance visual acuity (CDVA). Postoperative data included IOP, number of antiglaucoma medications, complications, and CDVA. For analysis purpose, two hypotensive medications combined in a single formulation were counted as two medications rather than a single medication.

\section{Trabecular Micro-Bypass Stent}

As a one-piece, L-shaped MIGS device, the iStent trabecular micro-bypass is made of heparin-coated biocompatible implant-grade titanium (nickel-free). The height and length of the iStent are $0.33 \mathrm{~mm}$ and $1.0 \mathrm{~mm}$, respectively. The device has a snorkel of $0.25 \mathrm{~mm}$ and $120 \mu \mathrm{m}$ in length and bore diameter, respectively, and the snorkel contains three retention arches to facilitate effective placement. The iStent enhances aqueous outflow is by producing a bypass through the trabecular meshwork and directly connecting the anterior chamber to Schlemm's canal. Upon implantation, the iStent's snorkel (i.e., the 
short side) remains in the anterior chamber while the device's body (i.e., the open half pipe lumen) sits within Schlemm's canal. The stent is contained within a stainless-steel, single-use inserter which is used in positioning the body and snorkel in the Schlemm canal and anterior chamber, respectively.

\section{Surgical Technique}

The first phase of the surgical procedure involved administering the patient with local anaesthesia and then sterilization. Following this, phacoemulsification was undertaken via a clear corneal temporal incision, after which the intraocular lens was inserted in the bag. In the event that the full dilation of the pupil was observed after implantation of the IOL; then intracameral acetylcholine $1 \%$ was used to achieve miosis Next, a viscoelastic substance was injected into the anterior chamber. The patient's head and microscope were repositioned and, in turn, gonio lens was used to view the angle providing a good view of the nasal trabecular meshwork. Without the gonio lens in place, the inserter containing a preloaded iStent was passed through the clear corneal temporal incision into the anterior chamber, then the gonio lens was applied again to observe the angle. The iStent device's leading edge was placed into the Schlemm canal in the superior nasal part of the trabecular meshwork, after which the inserter's button was pressed to release the stent. Following this, the inserter was extracted, the viscoelastic substance was washed away, the normal pressure was restored to the anterior chamber with a balanced salt solution, and the temporal phacoemulsification incision was closed. Finally, each patient received a sub-conjunctival injection of gentamicin and dexamethasone.

\section{Postoperative Medication and Follow-Up}

Glaucoma medications were discontinued for the patients postoperatively. Topical prednisolone acetate 1\% (Pred Forte; Allergan, Inc., Irvine, CA) was prescribed in tapering doses over 6 weeks starting with a drop every 2 hours and ending with a drop once daily. In addition, moxifloxacin (Vigamox; Alcon Laboratories, Inc., Fort Worth, TX) drops were prescribed to the patients 4 times daily for a period of 14 days. Follow-up appointments were scheduled with the patients at day one and two weeks after the surgery, as well as months $1,3,6,9,12,18,24$, and 36 . During the follow-up period, if IOP of greater than 18 $\mathrm{mmHg}$ was noticed, the surgeon increased medications accordingly to reach target IOP.

\section{Statistical Analysis}

CDVA was converted to logarithm of minimum angle of resolution (LogMAR). IOP spikes were defined as an elevation of IOP that was greater than or equal to 10 $\mathrm{mmHg}$ at any point following the surgical intervention. Efficacy analysis involved IOP and the number of medications received. Finally, safety evaluations involved the, the gain of Snellen lines of CDVA, and loss of Snellen lines of CDVA, the incidence of IOP spikes, complications, and the need for additional glaucoma surgery.

IBM SPSS v. 22 for the Windows operating system (IBM Corp, Armonk, NY, USA) was used to conduct statistical analysis. The Shapiro-Wilk test was utilized as the test of normality, and paired t-tests were used to compare the baseline and post-operative IOP values. CDVA and the number of glaucoma medications were comparatively examined using the Wilcoxon signed rank test. Additionally, the percent of eyes per number of medication was compared at baseline and at 12 months/last follow-up by chi-square test. Linear regression analysis was performed to predict future reduction in IOP. The cutoff level for a statistically significant result was 0.05 . In comparing the pre-operative data to last follow-up.

\section{Results}

In this interventional case series, 36 eyes of 33 patients (23 males, 10 females) underwent successful phacoemulsification with the implantation of an intraocular lens and a single trabecular micro-bypass stent (iStent) at a secondary hospital from June 2016 to June 2019. Three patients had bilateral significant cataract and concomitant glaucoma therefore both eyes were included in the analysis. All eyes have been followed out to 1 year post-surgery. Table 1 shows the demographic and baseline characteristics of the study population. The cohort comprised of primary open-angle glaucoma $(n=26)$, pseudoexfoliative glaucoma $(n=8)$, and pigmentary glaucoma $(n=2)$. The mean age of the patients was $66.34 \pm 6.12$ years. None of the eyes had previously underwent glaucoma surgery. All eyes had visually significant cataract, with $86.1 \%$ ( $n=31$ ) having baseline CDVA of 20/50 or worse. For the overall cohort, the baseline mean IOP and mean medications were $17.47 \pm 5.44 \mathrm{mmHg}$ and $2.69 \pm 0.92$, respectively. The mean cup-to-disc ratio was $0.64 \pm 0.17$.

Figures 1-3 provide an overview of the mean IOP, the mean number of glaucoma medications received, and the mean CDVA from baseline to the final follow-up for the entire 
Table I Demographics and Baseline Characteristics for the Overall Study Populations

\begin{tabular}{|l|l|}
\hline Parameters & Value (Mean \pm SD) \\
\hline Overall OAG & $\mathrm{n}=36$ \\
POAG & $\mathrm{n}=26$ \\
PXF & $\mathrm{n}=8$ \\
PG & $\mathrm{n}=2$ \\
Mean age (years) & $66.34 \pm 6.12$ \\
Gender (males/females) & $23 / 10$ \\
Cup-to-disc ratio & $0.64 \pm 0.17$ \\
Baseline number of medications & $2.69 \pm 0.92$ \\
Baseline IOP (mmHg) & $17.47 \pm 5.44$ \\
Baseline CDVA (logMAR (Snellen)) & $0.77 \pm 0.56(20 / 117)$ \\
Follow-up time (month) & $18.25 \pm 11.05$ \\
\hline
\end{tabular}

Abbreviations: IOP, intraocular pressure; $n$, number of eyes; CDVA, corrected distance visual acuity; OAG, open-angle glaucoma; POAG, primary open-angle glaucoma; PXF, pseudo exfoliative glaucoma; PG, pigmentary Glaucoma; SD, standard deviation.

cohort. The results indicate that IOP and CDVA improved immediately post-operatively and, after 14 days, stabilised. Overall the average glaucoma medication usage decreased compared to baseline.

\section{Efficacy}

An analysis of the data demonstrated a reduction in IOP and the number of anti-glaucoma medications used. In comparison to baseline, the mean IOP at the final followup decreased from $17.47 \pm 5.44$ to $13.44 \pm 1.99(23.1 \%$ reduction) $(\mathrm{P}<0.001)$. For future cases, the regression analysis estimated a reduction of IOP by $29.4 \%$ $(\mathrm{P}<0.001)$. In comparison to baseline, the mean medications at the final follow-up decreased from $2.69 \pm 0.92$ to $1.47 \pm 1.13(45.4 \%$ reduction $)(\mathrm{P}<0.001)$.

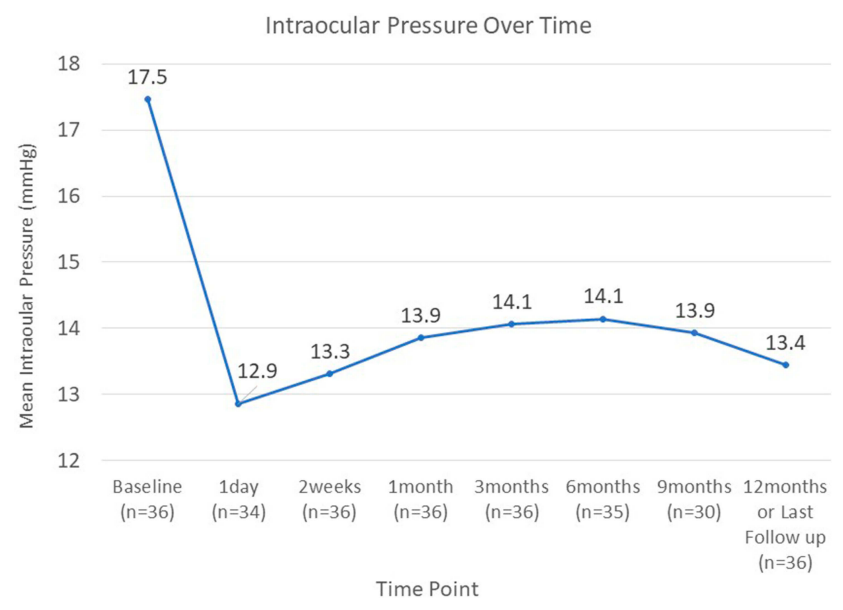

Figure I Intraocular pressure over time.
Pre-operatively, no eyes were medication-free, while at the final follow-up session, $27.8 \%(n=10)$ of eyes were medication-free. At baseline, $25 \%$ of eyes were receiving 4 medications, while only $5.6 \%$ were receiving same number at the final follow-up session. Another $25 \%$ of eyes were receiving 3 medications at baseline, which contrasted with 5.6\% at the final follow-up session. (Table 2).

A sub-analysis was performed on four eyes with starting IOP in the $8-10 \mathrm{mmHg}$ range who were receiving $2-4$ medications pre-operatively. The IOP values for these eyes at the final follow-up was in the $12-14 \mathrm{mmHg}$ range on 0-2 medications, in particular, 2 eyes were medication free.

\section{Safety}

In this study, 36 eyes underwent uneventful phacoemulsification cataract surgery with implantation of one iStent device. During the entire period of follow-up, no loss of light perception, sight-threatening disorders, choroidal detachment, retinal detachment, endophthalmitis, or hypotony maculopathy or stent-related complications were observed, including peripheral anterior synechiae, loss of stent, stent obstruction, or haemorrhage. In addition, no eyes needed to receive another glaucoma surgery during the follow-up period. Corrected distance visual acuity (CDVA) significantly improved from $0.77 \pm 0.56$ LogMAR at baseline to $0.19 \pm 0.23$ LogMAR at 12 months/last follow-up ( $\mathrm{p}<0.005$ ).

Transient findings were observed during the early postoperative period that are expected following phacoemulsification alone, including corneal edema, inflammation, and discomfort which were all mild and were resolved without sequelae. Eight (22.2\%) eyes displayed IOP spikes which were primarily attributed to the steroid postoperative regimen and were successfully treated with glaucoma medications with no sequelae. In 1 of the 8 eyes, transient high IOP was observed on the operative day and was attributed to the entrapment of the viscoelastic substance within the anterior chamber. This patient was treated with 3 topical medications and IOP decreased on the first postop day and was controlled through all follow-up sessions. At 12 months follow-up, IOP was $13 \mathrm{mmHg}$ on 2 medications, while pre-operatively the IOP was $19 \mathrm{mmHg}$ on 2 medications.

\section{Discussion}

The iStent trabecular micro-bypass is the first FDAapproved MIGS device. Its application in the treatment 


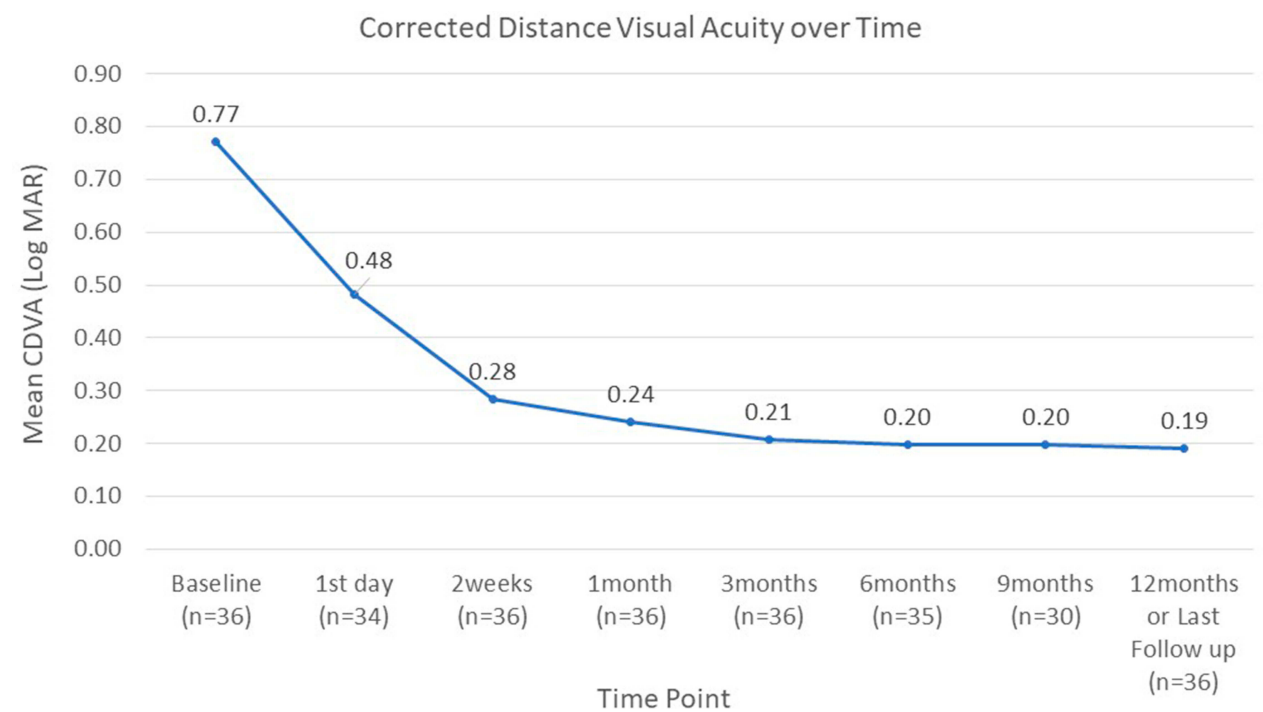

Figure 2 Corrected distance visual acuity over time.

of mild-to-moderate OAG has become increasingly prevalent. With the increasing use of this procedure, it is important to gather data which showcase patient outcomes in different populations and clinical settings. Therefore, due to the dearth of research on surgical outcomes for Middle East patients, the findings of this study will have considerable value for ophthalmological practitioners in comparable populations and clinical settings.

All patients included in this study were previously diagnosed with OAG and on an average of 2.69 topical medications. All patients also suffered from a visually significant cataract requiring extraction. Thus, by incorporating the iStent implantation into the conventional cataract removal procedure, close to one third of the eyes assessed

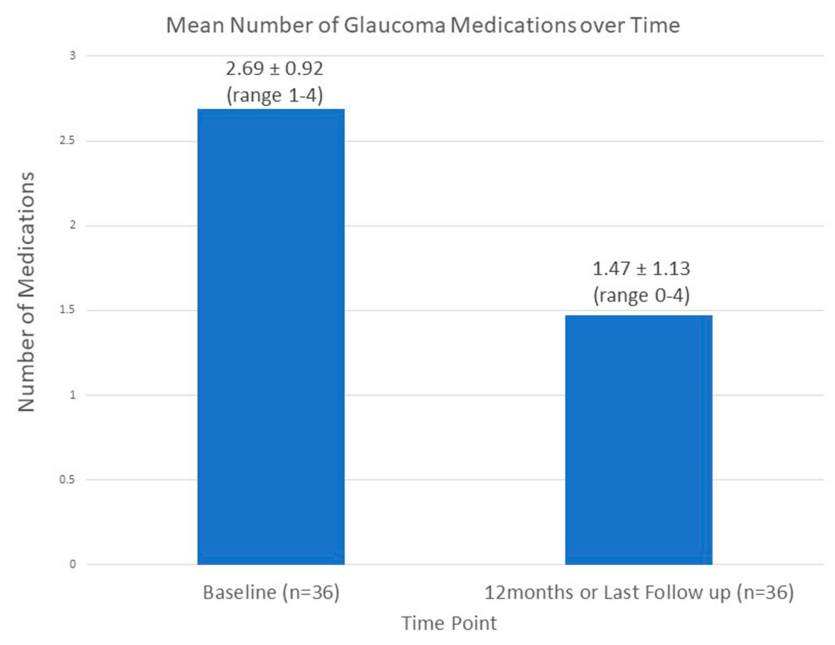

Figure 3 Mean number of glaucoma medication over time. in this study ceased to require medication by the final follow-up session (compared to $0 \%$ pre-operative) whilst $52.8 \%$ were receiving at least 2 fewer medications. The mean decrease in the number of medications was $45.4 \%$ (average 2.69 medications pre-surgery to 1.47 postsurgery; $\mathrm{P}<0.001)$. Mean IOP was significantly reduced by $4 \mathrm{mmHg}$, a $23.1 \%$ reduction from baseline $(\mathrm{P}<0.001)$. Several landmark studies have established the importance of even a $1 \mathrm{mmHg}$ decrease in IOP. The Early Manifest Glaucoma Trial showed a $10 \%$ lower risk of glaucoma progression for every $1 \mathrm{mmHg}$ IOP reduction. ${ }^{4}$ Indeed, a 4 $\mathrm{mmHg}$ reduction as demonstrated in this study is clinically relevant.

Several authors have published similar findings. ${ }^{17-20}$ As a case in point, Ferguson et al reported that after a period of 24 months, the mean IOP of patients who received an iStent declined from $19.13 \mathrm{mmHg}$ to $15.17 \mathrm{mmHg}$. This represents a decrease of $21 \%(\mathrm{P}<0.0001) .{ }^{20}$ Additionally, in the same study, the medication requirement for that cohort decreased by $49 \%(\mathrm{P}<0.0001)$. Other studies showed that the mean

Table 2 Percent of Eyes per Number of Medications at Baseline and 12 Months/Last Follow-Up

\begin{tabular}{|l|l|l|l|}
\hline AGM & Baseline & I 2 Months/Last Follow-Up & P-value \\
\hline 0 & $0 \%(0)$ & $27.8 \%(10)$ & \\
1 & $5.6 \%(2)$ & $13.9 \%(5)$ & \\
2 & $44.4 \%(16)$ & $47.25(17)$ & 0.010 \\
3 & $25 \%(9)$ & $5.6 \%(2)$ & \\
4 & $25 \%(9)$ & $5.6 \%(2)$ & \\
\hline
\end{tabular}

Abbreviation: AGM, anti-glaucoma medications. 
IOP reduction which resulted from iStent implantation ranged from $10-43 \% .^{21-23}$ When accounting for this wide range, the relationship between baseline IOP and post-operative IOP is noteworthy. It has been reported in the related literature that the percentage of the reduction in IOP is higher for patients who have a greater pre-operative IOP level. ${ }^{19}$ The differences in IOP reduction across studies can also be accounted for by the differences in study design (i.e. whether medications were washed out prior to conducting the surgical procedure and after surgery). In the presence of a washout, the pre-operative IOP for an eye is likely to be greater hence contributing to a greater degree of reduction. For illustration, Craven et al assessed the pre-operative mean IOP in the absence and presence of medication washout. They revealed that the IOP was $18.6 \mathrm{mmHg}$ and $25.4 \mathrm{mmHg}$, respectively. Furthermore, the researchers in the same study reported that the mean IOP at the 24-month follow-up was $17.1 \mathrm{mmHg}$ and the reduction without washout was significantly less than the reduction with washout ( $8 \%$ and $32.7 \%$, respectively). ${ }^{21}$ In the present study, the mean IOP reduction was statistically significant and although the baseline IOP was relatively low at $17.47 \mathrm{mmHg}$ without medication washout, the reduction in IOP falls in the middle of the range for the previously mentioned studies. $^{17-23}$

It is well recognized that topical glaucoma medications are associated with side effects and ocular surface damage along with the burden of medication costs and office visits and caregiver needs. In most instances, an increase in the number of medications results in a decline in medication adherence, a well know challenge with chronic medication usage especially in the setting of glaucoma. This can prevent achieving the consistent IOP reduction required to minimise the risk of disease progression. A lower medication burden may facilitate a more optimal adherence to medication. ${ }^{6,24-26}$ Moreover, dry eye is highly prevalent amongst the Saudi population ${ }^{27,28}$ and the use of antiglaucoma medication can cause further damage to the ocular surface. A reduction of topical anti-glaucoma medications after ab interno trabecular bypass surgery may improve the ocular surface. This would not only improve the symptoms of dry eye but also increases the success rate for further incisional glaucoma surgeries. ${ }^{11,29}$ It is noteworthy that $27.8 \%$ of eyes were medication-free and that the mean number of medications decreased by $45.4 \%$ from pre-operative representing a decrease in at least 1 medication.

Given the varying degrees of severity in cases of glaucoma, it is necessary to assess the results in the context of the goal of surgical treatment considering the pre-operative IOP, the number of medications and the target IOP. For instance, in eyes with well-controlled pre-operative IOP which is maintained with medication, the expected success may be considered as a stabilisation of IOP with a potential reduction in the number of medications. In contrast, for patients with uncontrolled IOP, the main expectation is likely to reduce IOP and together with a maintenance or reduction in the number of medications.

Importantly, the present study also demonstrated that eyes with a low medicated pre-operative IOP, ranging 8-10 $\mathrm{mmHg}$, are still able to achieve positive outcomes from the iStent procedure. In these eyes with already low IOP, medication reduction was achieved while still maintain pressures within target. Although the number of subjects in this subgroup was small $(n=4)$, these results are consistent with those of the earlier study conducted by Seibold et al. ${ }^{22}$

For the duration of the surveillance period, the safety profile of the present study was optimal. No patients were exposed to intra-operative or post-operative complications associated with the iStent procedure. Importantly, by the final follow-up session, none of the patients required further surgical intervention one year after stenting. Furthermore, each patient's conjunctiva was preserved. This is important in the event that further invasive interventions are necessary. The high safety profile of this study is consistent with other iStent studies reported in the literature. ${ }^{17,18,21,23,30,31}$

This high degree of efficacy and the safety results of iStent implantation combined with phacoemulsification could alter the algorithm for the management of glaucoma. The low complication rate and limited surgical time could encourage a surgeon to conduct an early surgical intervention, even as a preliminary step in the management of newly diagnosed OAG patients in the presence of cataract. At the same time, the patients will be encouraged to undergo surgery as it can decrease there need to employ topical medications with only an additional step to the conventional cataract surgery with limited complications.

Although the cost of the iStent surgery could be a concern to some patients and healthcare providers, many studies have found that, with quality of life gains, the iStent is a cost-efficient option to treat patients with mild to moderate open-angle glaucoma. ${ }^{25,26,32}$

Since no controls were used in this study (i.e. patients who underwent cataract removal without iStent implantation), it is not possible to separate the effects of cataract removal on the IOP reduction from that of the iStent which 
is one of the limitations of this study. Nevertheless, the mean reduction in IOP for this study's cohort (approximately $4 \mathrm{mmHg}$ ) was two times that of the anticipated reduction following phacoemulsification (approximately 1-2 $\mathrm{mmHg}$ ) which was reported by Vizzeri et al. ${ }^{33}$ Additionally, a case series conducted by Fea et $\mathrm{al}^{34}$ comparatively examined the IOP outcomes of two groups of patients. The study evaluated patients who underwent cataract extraction alone compared to those who underwent combined cataract extraction with iStent. The study found that the iStent group was associated with considerably lower numbers of medications and lower IOP values in addition to favourable long-term outcomes in the reduction of IOP. ${ }^{17}$

The lack of visual field tests and diurnal IOP assessments are limitations of the present study. Diurnal IOP assessments were not performed as they are not standard clinical assessments although are included in large randomized clinical trials. ${ }^{21}$ Additionally, as this is a real-world study, pre-operative medication washout was not conducted in the interests of preserving patient safety. In terms of possible avenues for further research, the literature would benefit from additional data to comparatively examine the impact of iStent in conjunction with phacoemulsification to the sole application of phacoemulsification. Another fruitful area to consider for further research is the comparison of the effects of iStent with those of other minimally-invasive glaucoma surgeries.

In conclusion, phacoemulsification with the implantation of iStent is an effective and safe procedure to lower IOP. Furthermore, it reduces the need for glaucoma medications, and assists in overcoming the problems associated with medication non-adherence in OAG patients. The iStent trabecular micro-bypass, a device with a high benefit to risk profile augments the existing treatment options for glaucoma. This is the first report of iStent usage in Saudi eyes demonstrating its effectiveness across patient populations.

\section{Author Details}

Ahmed AL Habash MD, Assistant Professor of Ophthalmology; Imam Abdulrahman Bin Faisal University, Dammam, Saudi Arabia; Glaucoma Consultant King Fahd Hospital of the University, Khobar, Saudi Arabia; Saudi Board of Ophthalmology; University of Dammam Ophthalmology Fellowship; International Council of Ophthalmology Fellowship; Former Fellow of Glaucoma and cataract at King Khalid Eye Specialist Hospital.

\section{Ethics}

The article was approved by the IRB committee of King Fahd Hospital of the University.

\section{Acknowledgment}

The authors thank Glaukos for funding this article's publication fee.

\section{Disclosure}

The authors did not receive any financial support from any public or private sources. The authors have no financial or proprietary interest in a product, method, or material described herein.

\section{References}

1. Quigley HA, Broman AT. The number of people with glaucoma world wide in 2010 and 2020. Br J Ophthalmol. 2006;90:262-267. doi:10.1136/bjo.2005.081224

2. Tabbara KF, Ross-Degnan D. Blindness in Saudi Arabia. JAMA. 1986;255:3378-3384. doi:10.1001/jama.1986.03370240048035

3. Cook C, Foster P. Epidemiology of glaucoma: what's new? Can J Ophthalmol. 2012;47(3):223-226. doi:10.1016/j.jcjo.2012.02.003

4. Leske MC, Heijl A, Hussein M, et al. Factors for glaucoma progression and the effect of treatment: the early manifest glaucoma trial. Arch Ophthalmol. 2003;121(1):48-56. doi:10.1001/archopht.121.1.48

5. Prum BE Jr, Rosenberg LF, Gedde SJ, et al. Primary open-angle glaucoma preferred practice pattern guidelines. Ophthalmology. 2016;123:P41-P111. doi:10.1016/j.ophtha.2015.10.053

6. Tsai JC. A comprehensive perspective on patient adherence to topical glaucoma therapy. Ophthalmology. 2009;116:S30-S36. doi:10.1016/j. ophtha.2009.06.024

7. Juzych MS, Chopra V, Banitt MR, et al. Comparison of long-term outcomes of selective laser trabeculoplasty versus argon laser trabeculoplasty in open-angle glaucoma. Ophthalmology. 2004;111 (10):1853-1859. doi:10.1016/j.ophtha.2004.04.030

8. Laser Trial Research Group G; Glaucoma Laser Trial Research Group. The Glaucoma Laser Trial (GLT) and glaucoma laser trial follow-up study: 7. Results. Am J Ophthalmol. 1995;120(6):718-731. doi:10.1016/S0002-9394(14)72725-4

9. Gedde SJ, Herndon LW, Brandt JD, Budenz DL, Feuer WJ, Schiffman JC. Tube Versus Trabeculectomy Study Group. Postoperative complications in the Tube Versus Trabeculectomy (TVT) study during five years of follow-up. Am $J$ Ophthalmol. 2012;153(5):804-814. doi:10.1016/j.ajo.2011.10.024

10. Saheb H, Ahmed IIK. Micro-invasive glaucoma surgery: current perspectives and future directions. Curr Opin Ophthalmol. 2012;23:96-104. doi:10.1097/ICU.0b013e32834ff1e7

11. Chen KH, Kim WI. Implantation of a CyPass supraciliary stent combined with targeted iStent trabecular microbypass stents in a phakic patient with primary open-angle glaucoma. $J$ Glaucoma. 2018;27(2):e37-e39. doi:10.1097/IJG.0000000000000835

12. Lynn SC FDA approves first glaucoma stent for use with cataract surgery. FDA Press Announcements [Internet]. Jun 2, 2012. [cited May 4, 2016]. Available from http://www.fda.gov/NewsEvents/ Newsroom/PressAnnouncements/ucm309667.htm.

13. Grant WM. Experimental aqueous perfusion in enucleated human eyes. Arch Ophthalmol. 1963;69(6):783-801. doi:10.1001/ archopht.1963.00960040789022 
14. Ethier CR, Kamm RD, Palaszewski BA, Johnson MC, Richardson TM. Calculations of flow resistance in the juxtacanalicular meshwork. Invest Ophthalmol Vis Sci. 1986;27(12):1741-1750.

15. Nichamin LD. Glaukos iStent ${ }^{\circledR}$ trabecular micro-bypass. Middle East Afr J Ophthalmol. 2009;16(3):138-140. doi:10.4103/0974-9233.56227

16. Fernandez-Barrientos Y, Garcia-Feijoo J, Martinez-de-la-Casa JM, et al. Fluorophotometric study of the effect of the glaukos trabecular microbypass stent on aqueous humor dynamics. Invest Ophthalmol Vis Sci. 2010;51(7):3327-3332. doi:10.1167/iovs.09-3972

17. Fea AM, Consolandi G, Zola M, et al. Micro-bypass implantation for primary open-angle glaucoma combined with phacoemulsification: 4-year follow-up. J Ophthalmol. 2015;2015:795357. doi:10.1155/ 2015/795357

18. Buffet J, Brasnu E, Baudouin C, Labb e A. Efficacy of 2 trabecular micro-bypass stents during phacoemulsification for mild to advanced primary open-angle glaucoma controlled with topical hypotensive medications. J Glaucoma. 2017;26(12):1149-1154. doi:10.1097/ IJG.0000000000000808

19. Ferguson TJ, Swan R, Ibach M, Schweitzer J, Sudhagoni R, Berdahl JP. Trabecular microbypass stent implantation with cataract extraction in pseudoexfoliation glaucoma. J Cataract Refract Surg. 2017;43(5):622-626. doi:10.1016/j.jcrs.2017.02.029

20. Ferguson TJ, Berdahl JP, Schweitzer JA, Sudhagoni RG. Clinical evaluation of a trabecular micro-bypass stent with phacoemulsification in patients with open-angle glaucoma and cataract. Clin Ophthalmol. 2016;10:1767-1773. doi:10.2147/OPTH.S114306

21. Craven ER, Katz LJ, Wells JM, Giamporcaro JE. Cataract surgery with trabecular micro-bypass stent implantation in patients with mild-to-moderate open-angle glaucoma and cataract: two-year follow-up. $J$ Cataract Refract Surg. 2012;38:1339-1345. doi:10.1016/j.jcrs.2012.03.025

22. Seibold LK, Gamett KM, Kennedy JB, et al. Outcomes after combined phacoemulsification and trabecular microbypass stent implantation in controlled open angle glaucoma. J Cataract Refract Surg. 2016;42:1332-1338. doi:10.1016/j.jcrs.2016.07.023

23. Neuhann TH, Hornbeak DM, Neuhann RT, Giamporcaro JE. Longterm effectiveness and safety of trabecular microbypass stent implantation with cataract surgery in patients with glaucoma or ocular hypertension: five-year outcomes. J Cataract Refract Surg. 2018;45 (3):312-320. doi:10.1016/j.jcrs.2018.10.029

24. Inoue K. Managing adverse effects of glaucoma medications. Clin Ophthalmol. 2014;8:903-913. doi:10.2147/OPTH.S44708
25. Patel V, Ahmed I, Podbielski D, Falvey H, Murray J, Goeree R. Costeffectiveness analysis of standalone trabecular micro-bypass stents in patients with mild-to-moderate open-angle glaucoma in Canada. J Med Econ. 2019;22(4):390-401. doi:10.1080/13696998.2019.1572013

26. Ngan K, Fraser E, Buller S, Buller A. A cost minimisation analysis comparing iStent accompanying cataract surgery and selective laser trabeculoplasty versus topical glaucoma medications in a public healthcare setting in New Zealand. Graefes Arch Clin Exp Ophthalmol. 2018;256(11):2181-2189.

27. Yasir ZH, Chauhan D, Khandekar R, Souru C, Varghese S. Prevalence and determinants of dry eye disease among 40 years and older population of Riyadh (except capital), Saudi Arabia. Middle East Afr J Ophthalmol. 2019;26(1):27-32. doi:10.4103/ meajo.MEAJO_194_18

28. Alshamrani AA, Almousa AS, Almulhim AA, et al. Prevalence and risk factors of dry eye symptoms in a Saudi Arabian population. Middle East Afr J Ophthalmol. 2017;24(2):67-73. doi:10.4103/ meajo.MEAJO 28116

29. Broadway DC, Grierson I, O’Brien C, Hitchings RA. Adverse effects of topical antiglaucoma medication: II. The outcome of filtration surgery. Arch Ophthalmol. 1994;112(11):1446-1454. doi:10.1001/ archopht.1994.01090230060021

30. Samuelson TW, Katz LJ, Wells JM, Duh Y-J, Giamporcaro JE. Randomized evaluation of the trabecular micro-bypass stent with phacoemulsification in patients with glaucoma and cataract. Ophthalmology. 2011;118:459-467. doi:10.1016/j.ophtha.2010.07.007

31. Chang DF, Donnenfeld ED, Katz LJ, et al. Efficacy of two trabecular micro-bypass stents combined with topical travoprost in open-angle glaucoma not controlled on two preoperative medications: 3-year follow-up. Clin Ophthalmol. 2017;11:523-528. doi:10.2147/OPTH. S121041

32. Iordanous Y, Hutnik CML, Malvankar M. Cost-effectiveness analysis of iStent and phacoemulsification versus glaucoma medications in the Ontario Health Insurance Plan (OHIP). Invest Ophthalmol Vis Sci. 2014;55(13):5595

33. Vizzeri G, Weinreb RN. Cataract surgery and glaucoma. Curr Opin Ophthalmol. 2010;21(1):20-24. doi:10.1097/ICU.0b013e328332f562

34. Fea AM. Phacoemulsification versus phacoemulsification with micro-bypass stent implantation in primary open-angle glaucoma: randomized double-masked clinical trial. J Cataract Refract Surg. 2010;36:407-412. doi:10.1016/j.jcrs.2009.10.031
Clinical Ophthalmology

\section{Publish your work in this journal}

Clinical Ophthalmology is an international, peer-reviewed journal covering all subspecialties within ophthalmology. Key topics include: Optometry; Visual science; Pharmacology and drug therapy in eye diseases; Basic Sciences; Primary and Secondary eye care; Patient Safety and Quality of Care Improvements. This journal is indexed on PubMed
Central and CAS, and is the official journal of The Society of Clinical Ophthalmology (SCO). The manuscript management system is completely online and includes a very quick and fair peer-review system, which is all easy to use. Visit http://www.dovepress.com/ testimonials.php to read real quotes from published authors.

\section{Dovepress}

